\title{
An inequality for longitudinal and transverse wave attenuation coefficients
}

\author{
Andrew N. Norri:** \\ Mechanical and Aerospace Engineering, Rutgers University, 98 Brett Road, Piscataway, NJ 08854
}

(Dated: September 24, 2018)

\begin{abstract}
Total absorption, defined as the net flux of energy out of a bounded region averaged over one cycle for time harmonic motion, must be non-negative when there are no sources of energy within the region. This passivity condition places constraints on the non-dimensional absorption coefficients of longitudinal and transverse waves, $\gamma_{L}$ and $\gamma_{T}$, in isotropic linearly viscoelastic materials. Typically, $\gamma_{L}, \gamma_{T}$ are small, in which case the constraints imply that coefficients of attenuation per unit length, $\alpha_{L}, \alpha_{T}$, must satisfy the inequality $\alpha_{L} / \alpha_{T} \geq 4 c_{T}^{3} / 3 c_{L}^{3}$ where $c_{L}, c_{T}$ are the wave speeds. This inequality, which as far as the author is aware, has not been presented before, provides a relative bound on wave speed in terms of attenuation, or vice versa. It also serves as a check on the consistency of ultrasonic measurements from the literature, with most but not all of the data considered passing the positive absorption test.
\end{abstract}

PACS numbers: 43.20.Gp, 43.40.Dx, 43.35.Gk, 43.20.Tb

\section{INTRODUCTION}

When an acoustic wave is incident on a passive obstacle, i.e. one with no active sources of energy present, the scattering process should not result in more energy than that of the incident wave itself. If the object is viscoelastic then the total energy should decrease by virtue of the passive absorbing properties of viscoelastic solids. The absorption of wave energy in solids has been considered from many points of view, ranging from its thermodynamic and molecular origins [1, Chs. 11-13], to subwavelength scattering effects at the crystalline and granular scale 2, Ch. 9], to physically consistent mathematical models 3, Chs. 3.E, 5.C-E] [4, Ch. 2]. The interest here is in linearly viscoelastic materials, and the implications of positive absorption, also known as passivity [5]. In particular, we derive a new and useful relation between the attenuation coefficients for longitudinal and transverse waves isotropic solids.

We begin in Section $\amalg$ with a background review of absorption and viscoelasticity theory. The main results are presented in Section III, where they are discussed in the context of published data on ultrasonic attenuation coefficients.

\section{BACKGROUND REVIEW}

\section{A. Absorption}

The energy lost in a passive target subject to an incident time harmonic acoustic wave is defined by the timeaveraged outward net flux over the bounding surface of

\footnotetext{
* norris@rutgers.edu
}

the object,

$$
\begin{aligned}
P_{\mathrm{abs}} & =-\int_{S}\langle p \mathbf{v}\rangle \cdot \mathrm{d} \mathbf{s} \\
& \geq 0 .
\end{aligned}
$$

Here, $p$ denotes acoustic pressure, $\mathbf{v}$ the particle velocity, $S$ is the enclosing surface, $\mathrm{d} \mathbf{s}=\mathbf{n} \mathrm{d} S$ is the surface element with the unit normal $\mathbf{n}$ outwards, and $\langle\cdot\rangle$ indicates the average over a period. To be specific consider a single solid object of volume $V$. Traction continuity across $S$ implies $-p \mathbf{n}=\boldsymbol{\sigma} \mathbf{n}$ where $\boldsymbol{\sigma}$ is the symmetric stress in the solid. Normal velocity is also continuous, and the divergence theorem therefore implies the equivalent definition

$$
P_{\mathrm{abs}}=\int_{V} \operatorname{div}\langle\boldsymbol{\sigma} \mathbf{v}\rangle \mathrm{d} V .
$$

\section{B. Viscoelasticity}

In order to accommodate a viscoelastic constitutive relation it is necessary to work with complex-valued quantities. The real and imaginary parts of material properties, such as density $\rho$ and elastic stiffness $\mathbf{C}$, are denoted in standard fashion using single and double primes:

$$
\rho=\rho^{\prime}+\mathrm{i} \rho^{\prime \prime}, \quad \mathbf{C}=\mathbf{C}^{\prime}+\mathrm{i} \mathbf{C}^{\prime \prime} .
$$

The density is also considered complex-valued as this represents a better alternative to using viscoelastic moduli in certain materials encountered especially in geophysical acoustics, such as poroelastic continua where the complex $\rho$ includes Darcy-like flow effects on the overall inertia 6, 7].

Notwithstanding the danger of confusion, we now let the field variables $\boldsymbol{\sigma}$ and $\mathbf{v}$ (until now assumed to be real quantities) denote complex-valued amplitudes with the time dependence $\mathrm{e}^{-\mathrm{i} \omega t}, \omega>0$, understood. The real physical quantities are $\operatorname{Re} \boldsymbol{\sigma}(\mathbf{x}) \mathrm{e}^{-\mathrm{i} \omega t}$ and $\operatorname{Re} \mathbf{v}(\mathbf{x}) \mathrm{e}^{-\mathrm{i} \omega t}$. 
With * denoting the complex-conjugate, (2) becomes

$$
P_{\mathrm{abs}}=\frac{1}{2} \operatorname{Re} \int_{V} \operatorname{div} \boldsymbol{\sigma} \mathbf{v}^{*} \mathrm{~d} V .
$$

Using the equation of motion,

$$
\operatorname{div} \boldsymbol{\sigma}+\mathrm{i} \omega \rho \mathbf{v}=0 .
$$

the absorption can be expressed

$$
P_{\text {abs }}=\frac{\omega}{2} \int_{V} \rho^{\prime \prime}|\mathbf{v}|^{2} \mathrm{~d} V+\frac{1}{2} \operatorname{Re} \int_{V} \operatorname{tr}\left(\boldsymbol{\sigma} \operatorname{grad} \mathbf{v}^{*}\right) \mathrm{d} V .
$$

Strain is the symmetric part of the displacement gradient $\varepsilon=\operatorname{Sym} \mathbf{U}, \mathbf{U}=\operatorname{grad} \mathbf{u}$, where $\mathbf{u}=(-i \omega)^{-1} \mathbf{v}$ is the displacement.

The viscoelastic linear constitutive relation between stress and strain in its most general form [4, Ch. 4] posits stress as a convolution of strain with a time dependent stiffness. The relation is then linear in the frequency domain,

$$
\boldsymbol{\sigma}=\mathbf{C} \boldsymbol{\varepsilon} \Leftrightarrow \sigma_{i j}=C_{i j k l} \varepsilon_{k l}
$$

with complex-valued stiffness $\mathbf{C}$ defined by the Fourier transform of the time-dependent moduli. The latter are assumed to have the symmetries associated with a symmetric strain and a symmetric stress, implying $C_{i j k l}=C_{i j l k}, C_{i j k l}=C_{j i k l}$. Hence, $\boldsymbol{\sigma}=\mathbf{C U}$, and $\operatorname{Retr}\left(\boldsymbol{\sigma} \operatorname{grad} \mathbf{v}^{*}\right)=-\omega \operatorname{Im} \operatorname{tr} \mathbf{U}^{*} \mathbf{C U}$. In purely elastic solids the stiffness $\mathbf{C}$ is real-valued and satisfies the usual symmetry in terms of the interchange of the "major indices" associated with a reversible strain energy function. This property does not extend to viscoelasticity. However, it is expected in the quasistatic limit, and it is therefore reasonable to assume that it holds for the real part of $\mathbf{C}$, at least in some range of frequencies, but is not valid for the imaginary part of $\mathbf{C}$. We therefore split the imaginary part into symmetric and anti-symmetric parts,

$$
\begin{aligned}
C_{i j k l}^{\prime} & =C_{k l i j}^{\prime} ; \quad \mathbf{C}^{\prime \prime}=\mathbf{C}_{S}^{\prime \prime}+\mathbf{C}_{A}^{\prime \prime}, \\
C_{S i j k l}^{\prime \prime} & =C_{S k l i j}^{\prime \prime}, \quad C_{A i j k l}^{\prime \prime}=-C_{A k l i j}^{\prime \prime} .
\end{aligned}
$$

The absorption can then be written

$$
P_{\text {abs }}=\frac{\omega}{2} \int_{V}\left(\rho^{\prime \prime}|\mathbf{v}|^{2}-\operatorname{tr} \mathbf{U}^{*} \mathbf{C}_{S}^{\prime \prime} \mathbf{U}\right) \mathrm{d} V .
$$

This is always non-negative if and only if $\rho^{\prime \prime}$ is nonnegative and $\mathbf{C}_{S}^{\prime \prime}$ is negative semi-definite in the sense that $\operatorname{tr} \mathbf{A}^{*} \mathbf{C}_{S}^{\prime \prime} \mathbf{A} \leq 0$ for all $\mathbf{A}=\mathbf{A}^{T} \neq 0$. In summary,

$$
P_{\mathrm{abs}} \geq 0 \Leftrightarrow \rho^{\prime \prime} \geq 0 \text { and } \mathbf{C}_{S}^{\prime \prime} \text { is negative semi-definite. }
$$

The absorption is identically zero if the density is purely real and $\mathbf{C}_{S}^{\prime \prime}$ vanishes. The latter condition is equivalent to the requirement that $\mathbf{C}$ is Hermitian, i.e. $C_{i j k l}=$ $C_{k l i j}^{*}$. Hence,

$$
P_{\text {abs }}=0 \Leftrightarrow \rho \text { is real and } \mathbf{C} \text { is Hermitian. }
$$

Note that the present results do not rely upon the necessary consequences of causality on the analytic properties of the complex-valued moduli, a topic that has been addressed well elsewhere, e.g. [8].

\section{Isotropic viscoelasticity}

The moduli have standard form with two complexvalued Lamé moduli, $\lambda$ and $\mu$,

$$
C_{i j k l}=\lambda \delta_{i j} \delta_{k l}+\mu\left(\delta_{i k} \delta_{j l}+\delta_{i l} \delta_{j k}\right) .
$$

This stiffness satisfies (8) with $\mathbf{C}_{A}^{\prime \prime}=0$, i.e. $\mathbf{C}$ is not Hermitian, and it is therefore expected that $P_{\text {abs }}$ will be non-zero. The absorption becomes

$$
P_{\mathrm{abs}}=\frac{\omega}{2} \int_{V}\left(\rho^{\prime \prime}|\mathbf{v}|^{2}-\kappa^{\prime \prime}|\operatorname{tr} \varepsilon|^{2}-\mu^{\prime \prime} \operatorname{tr}\left(\varepsilon_{d} \varepsilon_{d}^{*}\right)\right) \mathrm{d} V,
$$

where $\kappa=\lambda+\frac{2}{3} \mu$ is the bulk modulus and $\varepsilon_{d}=\varepsilon-\mathbf{I} \frac{1}{3} \operatorname{tr} \varepsilon$ is the deviatoric strain. Hence,

$$
P_{\text {abs }} \geq 0 \Leftrightarrow \rho^{\prime \prime} \geq 0, \kappa^{\prime \prime} \leq 0 \text { and } \mu^{\prime \prime} \leq 0 .
$$

This places constraints on the imaginary parts of the elastic moduli.

For instance, the Kelvin-Voigt model assumes that the stress is of the form

$$
\boldsymbol{\sigma}=\left(\lambda_{e}+\lambda_{v} \partial_{t}\right)(\operatorname{tr} \boldsymbol{\varepsilon}) \mathbf{I}+2\left(\mu_{e}+\mu_{v} \partial_{t}\right) \boldsymbol{\varepsilon}
$$

where $\lambda_{e}, \mu_{e}$ are the elastic moduli, $\lambda_{v}, \mu_{v}$ are generalized viscosities, all real quantities. Hence, $\lambda^{\prime \prime}=-i \omega \lambda_{v}$, $\mu^{\prime \prime}=-i \omega \mu_{v}$, and the constraints (14) are satisfied if $\lambda_{v}+\frac{2}{3} \mu_{v}>0$ and $\mu_{v}>0$.

Positive absorption has implications for other elastic moduli. For any complex-valued elastic modulus $M=M^{\prime}+\mathrm{i} M^{\prime \prime}$, the loss factor [9, p. 7] is defined as $d_{M}=-M^{\prime \prime} / M^{\prime}$, so that $M=\left(1-\mathrm{i} d_{M}\right) M^{\prime}$. The constraints (14) imply that $d_{\mu} \geq 0, d_{\kappa} \geq 0$. The longitudinal modulus, Poisson's ratio, Young's modulus and area modulus [10] are $L=\lambda+2 \mu, \nu=\lambda /[2(\lambda+\mu)]$, $E=2(1+\nu) \mu$ and $A=(1-\nu)^{-1}(1+\nu) \mu$, respectively. Their loss factors are, to leading order in $d_{\mu}$ and $d_{\kappa}$,

$$
\begin{aligned}
d_{A} & =c_{1} d_{\mu}+\left(1-c_{1}\right) d_{\kappa} \\
d_{L} & =\left(1-c_{1}\right) d_{\mu}+c_{1} d_{\kappa} \\
d_{E} & =c_{2} d_{\mu}+\left(1-c_{2}\right) d_{\kappa} \\
d_{\nu} & =\left(3 \nu^{\prime}\right)^{-1}\left(1+\nu^{\prime}\right)\left(1-2 \nu^{\prime}\right)\left(d_{\mu}-d_{\kappa}\right)
\end{aligned}
$$

where $c_{1}=\frac{1}{3}\left(1+\nu^{\prime}\right) /\left(1-\nu^{\prime}\right), c_{2}=\frac{2}{3}\left(1+\nu^{\prime}\right)$. Positive definiteness of the elastic strain energy requires that $-1<\nu^{\prime}<\frac{1}{2}$, and hence $0<c_{1}<c_{2}<1$ and the loss factors $d_{L}, d_{E}$ and $d_{A}$ are always non-negative with values between $d_{\mu}$ and $d_{\kappa}$. The Poisson's ratio loss factor may in principle be of either sign, although reported values, e.g. for rubber [8], are positive indicating $d_{\mu}>d_{\kappa}$. 


\section{ELASTIC WAVE DAMPING}

\section{A. Constraints on absorption coefficients}

The complex-valued longitudinal and transverse wavenumbers, $\tilde{k}_{L}$ and $\tilde{k}_{T}$, are

$$
\tilde{k}_{L}=\frac{\omega}{\tilde{c}_{L}}, \quad \tilde{k}_{T}=\frac{\omega}{\tilde{c}_{T}}, \quad \tilde{c}_{L}=\sqrt{\frac{\lambda+2 \mu}{\rho}}, \tilde{c}_{T}=\sqrt{\frac{\mu}{\rho}} .
$$

A common method for characterizing viscoelasticity is via ultrasonic measurement of the complex-valued wavenumbers. Specifically, we assume that the viscoelastic moduli are defined in terms of two real-valued wave speeds $c_{L}, c_{T}$ and two non-dimensional absorption coefficients $\gamma_{L}, \gamma_{T}$ :

$$
\tilde{k}_{M}=k_{M}\left(1+\mathrm{i} \gamma_{M}\right) \text { with } k_{M}=\frac{\omega}{c_{M}}, \quad M=L, T \text {. }
$$

According to this definition, $c_{M}=\left(\operatorname{Re} \tilde{c}_{M}^{-1}\right)^{-1}, M=$ $L, T$. It follows from the imaginary parts of the identities $\tilde{c}_{M}^{2}=c_{M}^{2} /\left(1+\mathrm{i} \gamma_{M}\right)^{2}, M=L, T$ that

$$
\begin{aligned}
\operatorname{Im} \frac{\mu}{\rho} & =-\frac{2 c_{T}^{2} \gamma_{T}}{\left(1+\gamma_{T}^{2}\right)^{2}} \\
\operatorname{Im} \frac{\kappa}{\rho} & =-\frac{2 c_{L}^{2} \gamma_{L}}{\left(1+\gamma_{L}^{2}\right)^{2}}+\frac{8 c_{T}^{2} \gamma_{T}}{3\left(1+\gamma_{T}^{2}\right)^{2}} .
\end{aligned}
$$

The three constraints of (14) imply that the left members in (19) are non-positive, and hence we obtain the main result of the paper:

$$
\gamma_{T} \geq 0, \quad \gamma \geq 0 ; \quad \gamma \equiv \frac{\gamma_{L}}{\left(1+\gamma_{L}^{2}\right)^{2}}-\frac{4 c_{T}^{2}}{3 c_{L}^{2}} \frac{\gamma_{T}}{\left(1+\gamma_{T}^{2}\right)^{2}}
$$

The first is usually satisfied because both $\gamma_{T}$ and $\gamma_{L}$ are specifically taken as non-negative. The condition for $\gamma$ places a constraint on $\gamma_{T}$ and $\gamma_{L}$ that depends upon the ratio of the undamped wave speeds. The value of $\gamma$ for measurements on Polymethylmethacrylate (PMMA) and other polymers are given in Table I], all satisfying the condition $\gamma>0$.

In practice the values of $\gamma_{L}$ and $\gamma_{T}$ are small, so that (20) $2(\gamma \geq 0)$ can be safely replaced by

$$
\frac{\gamma_{L}}{\gamma_{T}} \geq \frac{4 c_{T}^{2}}{3 c_{L}^{2}} \text { for } \gamma_{L}, \gamma_{T} \ll 1
$$

Thus, the ratio of the wave absorption factors must satisfy a strict but simple inequality when the attenuation is small. Note that the parameter depends upon the real part of the Poisson's ratio,

$$
\frac{4 c_{T}^{2}}{3 c_{L}^{2}}=\frac{2\left(1-2 \nu^{\prime}\right)}{3\left(1-\nu^{\prime}\right)}
$$

\begin{tabular}{lcccccc}
\hline \hline Material & Source & $c_{L}$ & $c_{T}$ & $\gamma_{L}$ & $\gamma_{T}$ & $\gamma$ \\
\hline PMMA & {$[11]$} & 2690 & 1340 & 0.0035 & 0.0053 & 0.0017 \\
Polyethylene & {$[11]$} & 2430 & 950 & 0.0073 & 0.0220 & 0.0028 \\
Phenolic polymer & {$[12]$} & 2840 & 1320 & 0.0119 & 0.0255 & 0.0045 \\
\hline \hline
\end{tabular}

TABLE I: An example of some absorption coefficients and the associated value of $\gamma$ from eq. (20). The numerical values from [11] are for measurements at room temperature of $\alpha \lambda=2 \pi \gamma$, the attenuation per wavelength in $\mathrm{dB}$, and uses the relation [13] $\alpha \lambda(\mathrm{dB})$ $=40 \pi \gamma / \ln 10$. Those of [12] are based on measurements of the attenuation coefficients $\alpha_{L}$ and $\alpha_{L}(\mathrm{~dB} / \mathrm{cm})$ at $25^{\circ} \mathrm{C}$ and frequency $1.8 \mathrm{MHz}$. Speeds are in $\mathrm{m} / \mathrm{s}$.

\section{B. Constraints on attenuation coefficients}

The amplitude of either wave type decays as $e^{-k_{M} \gamma_{M} x}$, $M=L$ or $T$. Attenuation as measured in $\mathrm{dB} / \mathrm{cm}$, for instance, defines the logarithm of the amplitude, and is therefore equivalent to measurement of $\alpha_{M} \equiv k_{M} \gamma_{M}$, $M=L$ or $T$, since $\alpha=k \gamma 20 / \ln 10$ where $k$ is wavenumber in $\mathrm{cm}^{-1}$. The multiplicative factor is irrelevant if we are only concerned with the ratio of the two attenuations. In the small attenuation regime eq. (21) then implies

$$
\frac{\alpha_{L}}{\alpha_{T}} \geq \frac{4 c_{T}^{3}}{3 c_{L}^{3}}
$$

\begin{tabular}{ccccccc}
\hline \hline Material & $f$ & $c_{L}$ & $c_{T}$ & $\alpha_{L}$ & $\alpha_{T}$ & Eq. (23) \\
& MHz & m/sec & m/sec & dB/cm & dB/cm & \\
\hline PMMA & 6 & 2756.4 & 1401.5 & 4.97 & 13.64 & $\checkmark$ \\
PMMA & 10 & 2760.5 & 1404.8 & 7.69 & 23.99 & $\checkmark$ \\
PMMA & 18 & 2764.2 & 1405.1 & 12.68 & 37.21 & $\checkmark$ \\
PMMA & 20 & 2765.1 & 1405.7 & 12.64 & 44.28 & $\checkmark$ \\
PMMA & 30 & 2765.5 & 1406.1 & 19.64 & 63.94 & $\checkmark$ \\
polymer \#1 & 1.8 & 2180 & 1080 & 1.4 & 6.7 & $\checkmark$ \\
polymer \#2 & 1.8 & 2040 & 830 & 1.8 & 15 & $\checkmark$ \\
\hline \hline
\end{tabular}

TABLE II: $f=\omega / 2 \pi$. Velocity and attenuation data for PMMA at $22.2^{\circ} \mathrm{C}$ and atmospheric pressure [14]. The data for the other polymers are from [15], where polymer \#1 is poly (4-methyl pentene-1) and polymer \#2 is poly(acrylonitrile-butadiene-styrene).

We consider the passivity constraint (23) in light of some reported ultrasonic data [14, 15] in Table II] The values of absorption indicates loss moduli of $1 \%$ or less than the real parts, i.e. small attenuation for which the criterion (23) applies, and is met for the data in Table II. Velocity and attenuation data for styrene-butadiene 
rubber [16] at $1 \mathrm{MHz}$ over a temperature range from $0^{\circ}$ to $20^{\circ}$ is consistent with (23).

Laymen et al. [17] provide curve-fitted equations, eqs. (15-18) in [17], for all four of the parameters in (23) based on ultrasonic measurements on a particulate composite sample over a broad frequency range (2 to $10 \mathrm{MHz}$ ). It may be easily verified that these wave speeds and attenuations satisfy the condition (23) over the entire range of frequencies considered. Measurements of high frequency (25 to $65 \mathrm{MHz}$ ) velocities and attenuation in passive ma- terials for ultrasonic transducers at room temperature are given in 18]. The materials include alumina/EPO-TEK 301 composites and tungsten/EPOTEK 301 composites. We have checked that all of the parameters reported satisfy eq. (23). Pinton et al. 19] measured attenuation and absorption of ultrasound in skull bone. They reported longitudinal absorption of $2.7 \mathrm{~dB} / \mathrm{cm}$ and shear absorption of $5.4 \mathrm{~dB} / \mathrm{cm}$ at the assumed longitudinal and shear wave speeds of $3,000 \mathrm{~m} / \mathrm{s}$ and $1,500 \mathrm{~ms}$, respectively, which clearly satisfies the positive absorption condition (23).

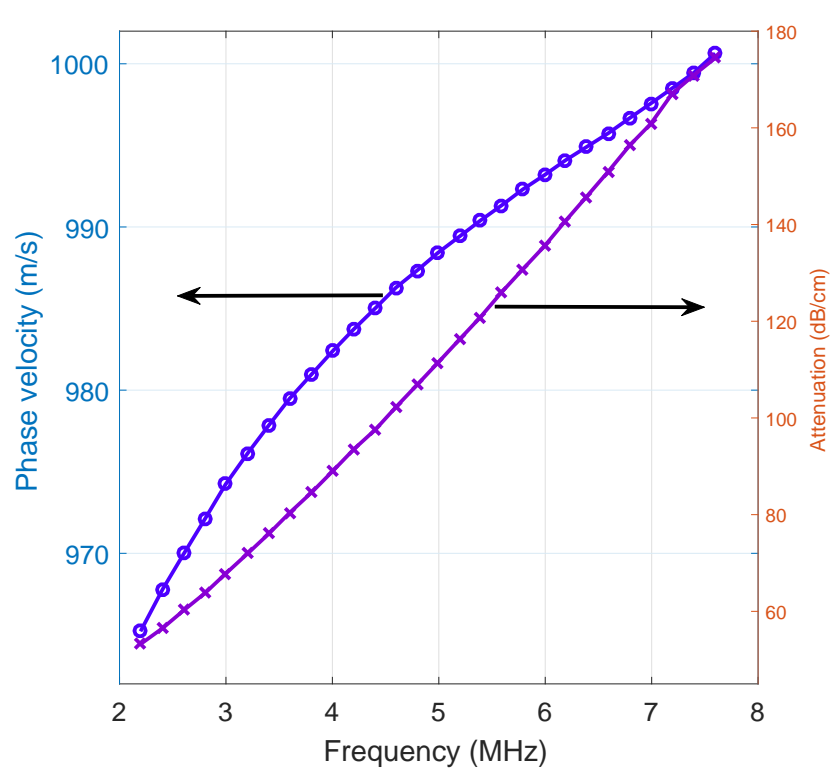

FIG. 1: Phase velocity and attenuation as functions of frequency for a high-density polyethylene sample: longitudinal (left) and transverse (right). Data from [20, Figure 4].

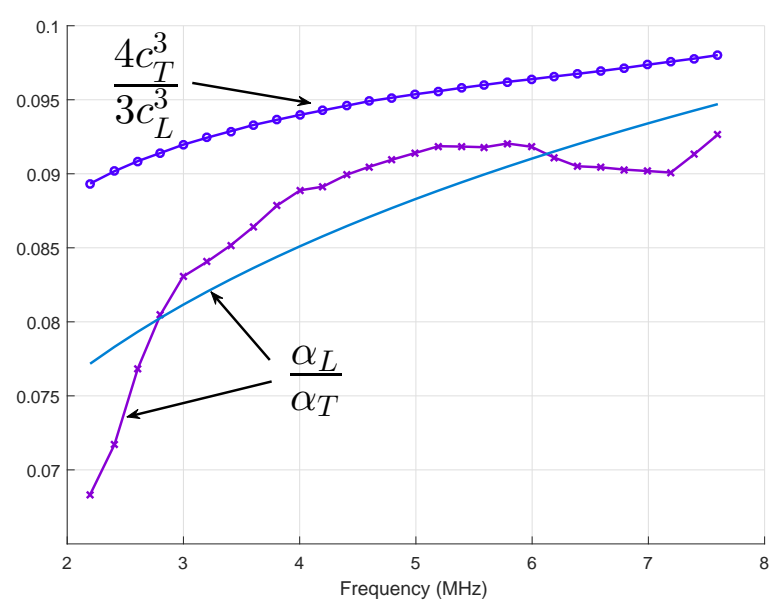

FIG. 2: The two terms in the passivity inequality (23) calculated from the data of Figure 1] The continuous curve for $\alpha_{L} / \alpha_{T}$ is based on eq. (24) and Table III.

As a final example we consider the velocity and at- tenuation data shown in Figure 11 which reproduces as accurately as possible the measurements reported in 20] for a high-density polyethylene sample. The calculated values of the two terms in eq. (23) are plotted in Figure 2. The smooth curve for $\alpha_{L} / \alpha_{T}$ uses a fitted power law model for attenuation proposed by Szabo and $\mathrm{Wu}$ 21] and applied to the data of $\mathrm{Wu}$ 20]. The model assumes the attenuation has frequency dependence of the form

$$
\alpha_{M}=\alpha_{0 M}+\alpha_{1 M}|f|^{y_{M}}, \quad M=L, T,
$$

where the coefficients for the high-density polyethylene sample are in Table III. Note that the formula corresponding to eq. (24) in Szabo and Wu [21] has $|\omega|$ instead of $|f|$, but we find that the numbers reported there are for eq. (24).

It is evident from the relative positions of the curves in Figure 2 that the passivity inequality (23) is not satisfied at any of the frequencies considered. We note that eq. (23) is an approximation valid for small values of attenuation. The precise condition $\gamma \geq 0$ may be written in 


\begin{tabular}{cccccccc}
\hline \hline$c_{L}$ & $c_{T}$ & $\alpha_{0 L}$ & $\alpha_{1 L}$ & $y_{L}$ & $\alpha_{0 T}$ & $\alpha_{1 T}$ & $y_{T}$ \\
\hline 2380 & 987 & 0 & 1.522 & 1.171 & -0.517 & 22.80 & 1.00 \\
\hline \hline
\end{tabular}

TABLE III: Absorption coefficients $\alpha_{0 M}(\mathrm{~dB} / \mathrm{cm}), \alpha_{1 M}$ $\left(\mathrm{dB} /(\mathrm{MHz})^{y_{\mathrm{M}}}-\mathrm{cm}\right)$, and exponential powers $y_{\mathrm{M}}$ for high density polyethylene. Speeds, in $\mathrm{m} / \mathrm{s}$, are for the reference frequency 4.8 MHz. Data from 21] except for $\alpha_{0 T}$ which is not given there but is found here by least square fitting, and $y_{T}$ which is found to provide better accuracy for $\alpha_{T}$ than the value 0.95 in [21].

similar form as

$$
\frac{\alpha_{L}}{\alpha_{T}} \geq \frac{4 c_{T}^{3}}{3 c_{L}^{3}} I \text { where } I=\left(\frac{1+\gamma_{L}^{2}}{1+\gamma_{T}^{2}}\right)^{2}
$$

Generally, the factor $I$ is close to but slightly less than unity, with $0.9964 \leq I \leq 0.9970$ for the data of Figure 1. The effect of including this term in Figure 2 is almost imperceptible, i.e., the data is in violation of the passivity condition for the bulk modulus. We can only conclude that the attenuation data for high-density polyethylene is not consistent with a passive linear viscoelastic model with frequency dependent complex-valued density and elastic moduli.

We note, however, that for the other data sets reported in [20], for samples of low-density polyethylene and Lexan Plexiglas, we find the passivity condition (23) is satisfied.

\section{CONCLUSION}

The main finding is the constraint on the nondimensional absorption parameter $\gamma$ in Eq. (20). For given values of wave speeds and shear absorption $\gamma_{T}$ this sets a lower bound on the longitudinal absorption $\gamma_{L}$. The inequality $\gamma \geq 0$ has direct interpretation when absorption is small $\left(\gamma_{L}, \gamma_{T} \ll 1\right)$, implying that the ratio of the attenuations per unit length, $\alpha_{L} / \alpha_{T}$, has a lower bound that depends on the ratio of the wave speeds, Eq. (23). The lower bound tends to zero as the Poisson's ratio of the material tends to $\frac{1}{2}$ ( $\Leftrightarrow c_{T} / c_{L} \rightarrow 0$ ). For instance, measurements of ultrasonic properties of soft tissues and tissue-like materials 22] shows shear wave attenuation coefficients on the order of $10^{4}$ times the longitudinal wave attenuation coefficients. Equation (23) then implies, under the small absorption assumption, that the transverse wave speed must be less than $4.2 \%$ the value of the longitudinal speed.
[1] A.B. Bhatia. Ultrasonic Absorption: An Introduction to the Theory of Sound Absorption and Dispersion in Gases, Liquids and Solids. Oxford University Press, 1967.

[2] Lester W. Schmerr, Jr. Fundamentals of Ultrasonic Nondestructive Evaluation. Springer, second edition, 2016.

[3] B. A. Auld. Acoustic Fields and Waves in Solids, Vol. I. Wiley Interscience, New York, 1973.

[4] José M. Carcione. Wave Fields in Real Media: Wave Propagation in Anisotropic, Anelastic, and Porous Media. Pergamon, 2001.

[5] Richard L. Weaver and Yih-Hsing Pao. Dispersion relations for linear wave propagation in homogeneous and inhomogeneous media. J. Math. Phys., 22(9):1909-1918, 1981.

[6] David Linton Johnson, Joel Koplik, and Roger Dashen. Theory of dynamic permeability and tortuosity in fluidsaturated porous media. Journal of Fluid Mechanics, 176(-1):379, mar 1987.

[7] Bastien Dupuy and Alexey Stovas. Influence of frequency and saturation on AVO attributes for patchy saturated rocks. Geophysics, 79(1):B19-B36, jan 2014.

[8] T. Pritz. Frequency dependences of complex moduli and complex Poissons ratio of real solid materials. J. Sound. Vib., 214(1):83-104, Jul 1998.

[9] B. E. Read and G. D. Dean. Determination of Dynamic Properties of Polymers and Composites. Wiley, 1978.

[10] N. H. Scott. An area modulus of elasticity: Definition and properties. J. Elasticity, 58:269-275, 2000.

[11] Bruce Hartmann and Jacek Jarzynski. Ultrasonic hysteresis absorption in polymers. J. Appl. Phys., 43(11):4304-4312, 1972.
[12] Bruce Hartmann. Ultrasonic properties of phenolic and poly(phenylquinoxaline) polymers. J. Appl. Polym. Sci., 19(12):3241-3255, Dec 1975.

[13] Luise S. Schuetz and Werner G. Neubauer. Acoustic reflection from cylinders - nonabsorbing and absorbing. $J$. Acoust. Soc. Am., 62(3):513-517, 1977.

[14] James R. Asay, Donald L. Lamberson, and Arthur H. Guenther. Pressure and temperature dependence of the acoustic velocities in polymethylmethacrylate. J. Appl. Phys., 40(4):1768-1783, 1969.

[15] Bruce Hartmann. Ultrasonic properties of poly(4-methyl pentene-1). J. Appl. Phys., 51(1):310-314, 1980.

[16] Yasaku Wada, Ryoichi Ito, and Hazime Ochiai. Comparison between mechanical relaxations associated with volume and shear deformations in styrene-butadiene rubber. J. Phys. Soc. Jpn., 17(1):213-218, Jan 1962.

[17] Christopher Layman, N. Sanjeeva Murthy, Ruey-Bin Yang, and Junru Wu. The interaction of ultrasound with particulate composites. J. Acoust. Soc. Am., 119(3):1449-1456, 2006.

[18] Haifeng Wang, T. Ritter, Wenwu Cao, and K.K. Shung. High frequency properties of passive materials for ultrasonic transducers. IEEE Trans. Ultrason. Ferroel. Freq. Cont., 48(1):78-84, 2001.

[19] Gianmarco Pinton, Jean-Francois Aubry, Emmanuel Bossy, Marie Muller, Mathieu Pernot, and Mickael Tanter. Attenuation, scattering, and absorption of ultrasound in the skull bone. Medical Physics, 39(1):299-307, 2012.

[20] Junru Wu. Determination of velocity and attenuation of shear waves using ultrasonic spectroscopy. J. Acoust. 
Soc. Am., 99(5):2871-2875, 1996.

[21] Thomas L. Szabo and Junru Wu. A model for longitudinal and shear wave propagation in viscoelastic media. $J$. Acoust. Soc. Am., 107(5):2437-2446, 2000.
[22] Ernest L. Madsen, H. John Sathoff, and James A. Zagzebski. Ultrasonic shear wave properties of soft tissues and tissuelike materials. J. Acoust. Soc. Am., 74(5):13461355, 1983. 